\title{
H- Injection Simulations for Multi-MW Upgrade (PIP-III)
}

\section{Matthew Hoppesch, Univ. of Illinois at Urbana-Champaign - SIST Intern I Jeffery Eldred and David Neuffer, FermiLab}

\section{Motivation}

The Fermilab Long-Baseline Neutrino Detectors will benefit from production of a higher power proton beam. Two proposals are being considered for PIP-III: A 2 GeV Rapid Cycling Synchrotron (RCS) and an $8 \mathrm{GeV}$ Superconducting Radio Frequency Linac (MI-LINAC). Designed to achieve a power of $2.4 \mathrm{MW}$, both proposals are demanding of $\mathrm{H}$ - injection. The goal of this research was to simulate and optimize injection for these designs. Typically, injection is optimized when the stripping foil has minimal interactions with the already

circulating beam. Too many foil interactions will eventually lead to foil mechanical failure as well as scattering losses.

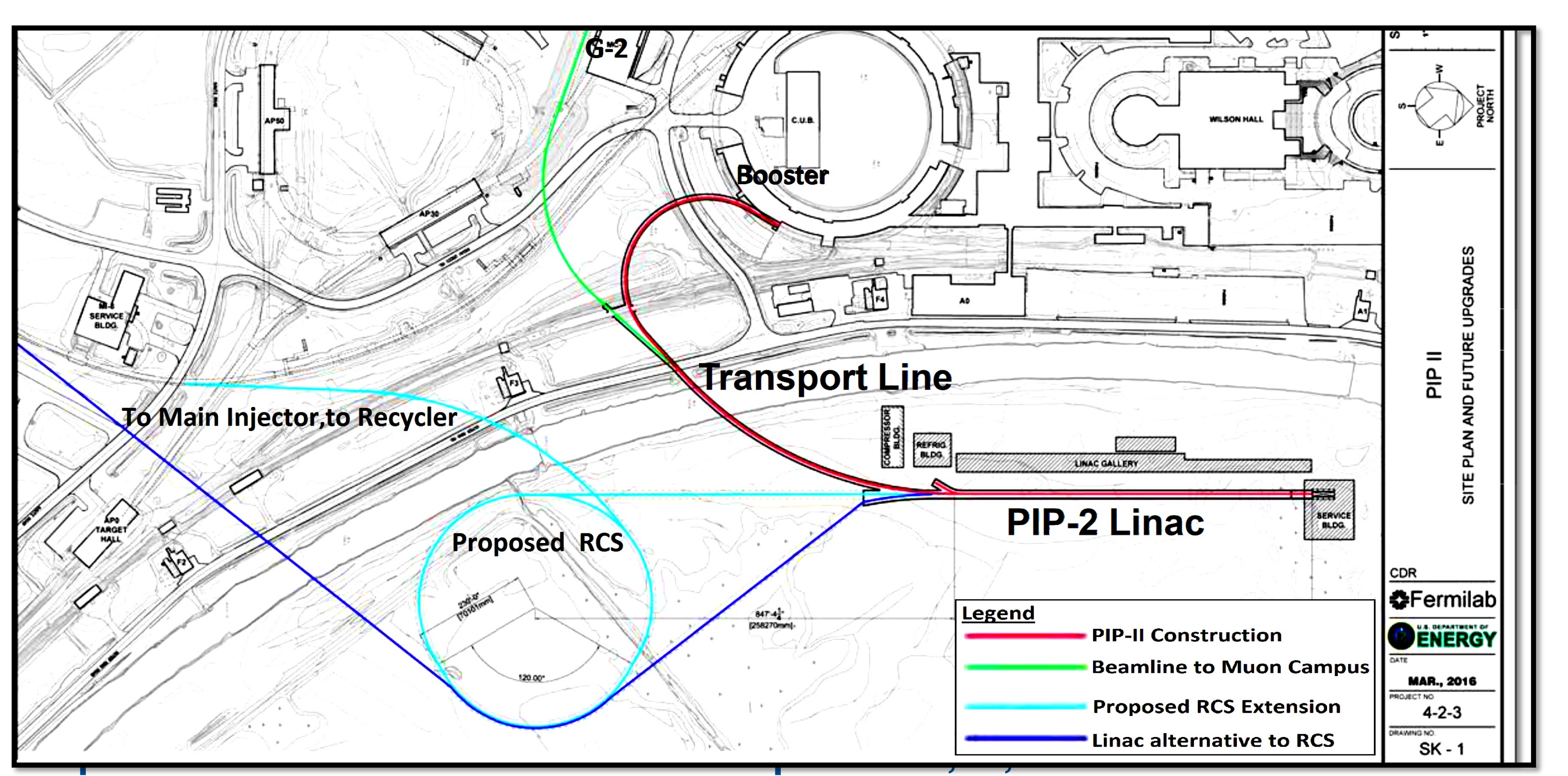

Potential locations of PIP 3 accelerators within the Fermilab complex

\section{Methods}

The simulation was written in Python, with extensive use of NumPy and Numba packages for efficient data handling and processing. It utilizes a transfer matrix to create a "one turn map" for particle tracking. The simulation recorded new particles entering the foil (primary hits), as well as already circulating particles interacting with it (secondary hits). It is modeled on a MathCad simulation, by Fermilab's Valeri Lebedev, which was created to simulate $\mathrm{H}$ - injection in the PIPII Conceptual Design Report (CDR). The PIP-II injection region includes dispersion, which required implementing longitudinal simulations. However, the RCS and MI-Linac propose injecting into dispersive free regions, so a 4D simulation was sufficient. Scans over many simulations were used frequently to optimize injection parameters. Investigations included partially implementing beam effects such as chromaticity, space charge, and transverse coupling, but none of these are included in the final simulation.

This manuscript has been authored by Fermi Research Alliance, LLC under Contract No. DE-AC02-07CH11359

\section{Timeline}

First - Benchmarked new program against PIP-II CDR

Next - Evaluated Foil Transversals for PIP-II on-momentum injection, as proposed by Paul Derwent.

Then - Adjusted simulation to needs of PIP-III simulation:

Higher Power beams need longer injection times, which required more efficient programming to handle

PIP-II will utilize an anti-correlated beam painting scheme, but for PIP-III correlated painting was investigated

Finally - Simulated several different PIP-III scenarios

Simulation Parameters

\begin{tabular}{|c|c|c|c|}
\hline Parameter & PIP-II & RCS & MI-Linac \\
\hline Energy & $0.8 \mathrm{GeV}$ & $2.0 \mathrm{GeV}$ & $8.0 \mathrm{GeV}$ \\
\hline Linac Current & $2 \mathrm{~mA}$ & $2 \mathrm{~mA} / 5 \mathrm{~mA}$ & $2 \mathrm{~mA}$ \\
\hline Intensity & $6.5 \mathrm{e} 12$ & 36 e12 & $5 \times 36 \mathrm{e} 12$ \\
\hline Pulse Duration & $0.52 \mathrm{~ms}$ & $5 \times .576 \mathrm{~ms} / 1.15 \mathrm{~ms}$ & $5 \times 2.9 \mathrm{~ms}$ \\
\hline Revolutions & & $5 \times 286 / 573$ & $5 \times 260$ \\
\hline $95 \%$ Norm Emit & $16 \mathrm{~mm} \mathrm{mrad}$ & $24 \mathrm{~mm} \mathrm{mrad}$ & $24 \mathrm{~mm} \mathrm{mrad}$ \\
\hline & $20.03 \mathrm{~m}$ & $33.00 \mathrm{~m}$ & $70.00 \mathrm{~m}$ \\
\hline & $6.17 \mathrm{~m}$ & $24.00 \mathrm{~m}$ & $40.00 \mathrm{~m}$ \\
\hline painting offset $x$ & $0.61 \mathrm{~cm}$ & $1.28 \mathrm{~cm}$ & $1.15 \mathrm{~cm}$ \\
\hline painting offset $_{y}$ & $1.10 \mathrm{~cm}$ & $1.51 \mathrm{~cm}$ & $0.85 \mathrm{~cm}$ \\
\hline$\beta_{\text {Linac }} / \beta_{\text {Ring }}$ & 0.48 & 0.20 & 0.20 \\
\hline Macro Particles & $1.75 \mathrm{e} 5$ & $1.43 \mathrm{e} 5$ & $1.30 \mathrm{e} 5$ \\
\hline
\end{tabular}

Secondary Foil Interactions are dependent on four factors: Correlated or Anti Correlated - Anticorrelated painting will generally accumulate more hits, but it eliminates protons with large action in both transverse planes.

Number of Revolutions - The more turns the Linac injects over, the greater interaction chance for an already circulating proton.

Painting Offset - A larger painting offset moves the foil further out of the circulating beam every turn.

Foil Size - A smaller foil decreases the interaction probability by presenting a smaller target.

Painting Schemes

Correlated (left) Anticorrelated (right)

\section{Results}

\begin{tabular}{|l||r|r|r|r|}
\hline Simulation & Hits $/ p^{+}$ & Pk. Hits $/ p^{+} \mathrm{cm}^{2}$ & Inj. Pt. $/ \mathrm{p}^{+} \mathrm{cm}^{2}$ & $\left\langle\right.$ Hits $\left./ \mathrm{p}^{+} \mathrm{ms}\right\rangle$ \\
\hline PIP-II CDR & 6.98 & 60.6 & $14.0 / 28.9$ & 13.5 \\
PIP-II on-p & 10.60 & 64.7 & $20.7 / 35.4$ & 20.5 \\
\hline 5 mA RCS CO & 4.2 & 67.0 & $19.9 / 47.8$ & 3.69 \\
5 mA RCS AC & 6.90 & 60.6 & $28.3 / 56.0$ & 6.00 \\
2 mA RCS CO & 9.73 & 172.1 & $55.6 / 83.5$ & 3.38 \\
2 mA RCS AC & 16.92 & 150.8 & $78.3 / 105.2$ & 5.88 \\
\hline MI-Linac CO & 9.76 & 269.6 & $108.7 / 213.9$ & 0.67 \\
MI-Linac AC & 18.70 & 262.6 & $137.7 / 243.4$ & 1.29 \\
\hline
\end{tabular}
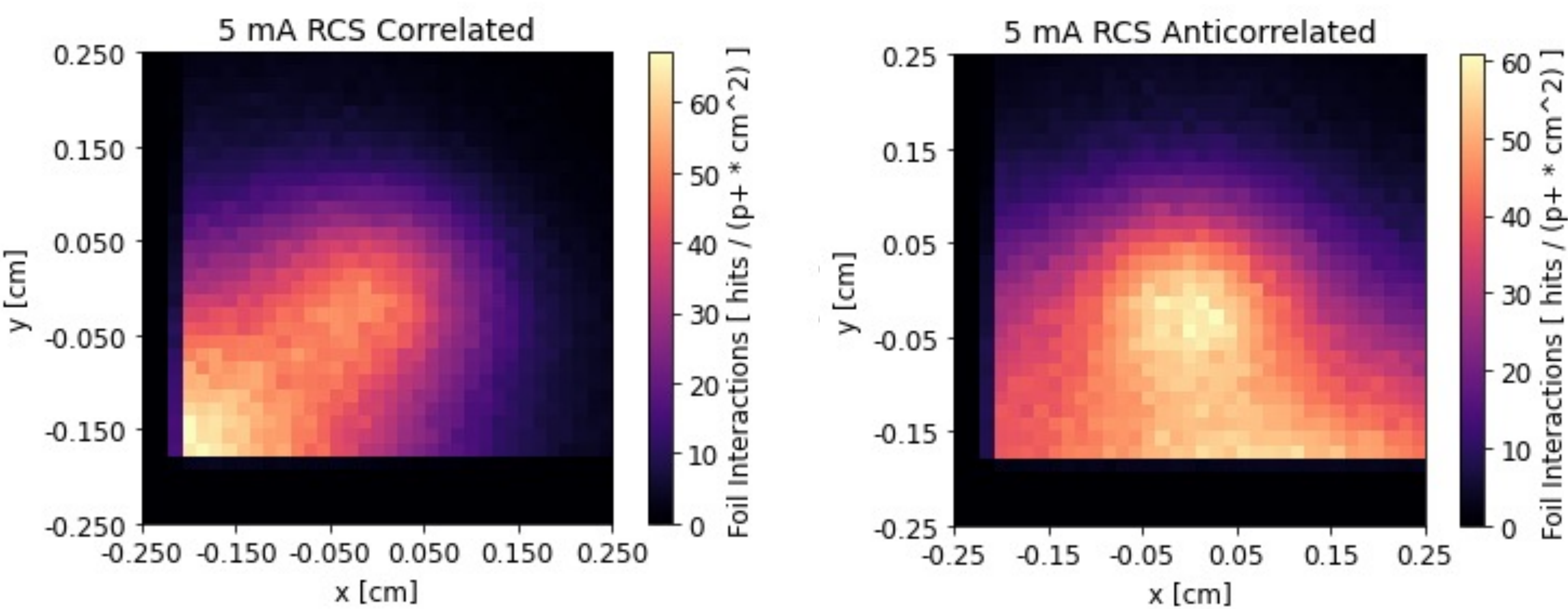

Hit density distribution at foil for Correlated (left) and Anticorrelated (right)

Each PIP-III scenario accumulates fewer total hits with a correlated painting scheme. However, the anticorrelated scheme does marginally reduce the peak hits density. Dispersion in the injected region were shown to move the anticorrelated peak hit density to the corner. (PIP-II) Also, a PIP-II on-momentum injection scheme seems to increase foil interactions.
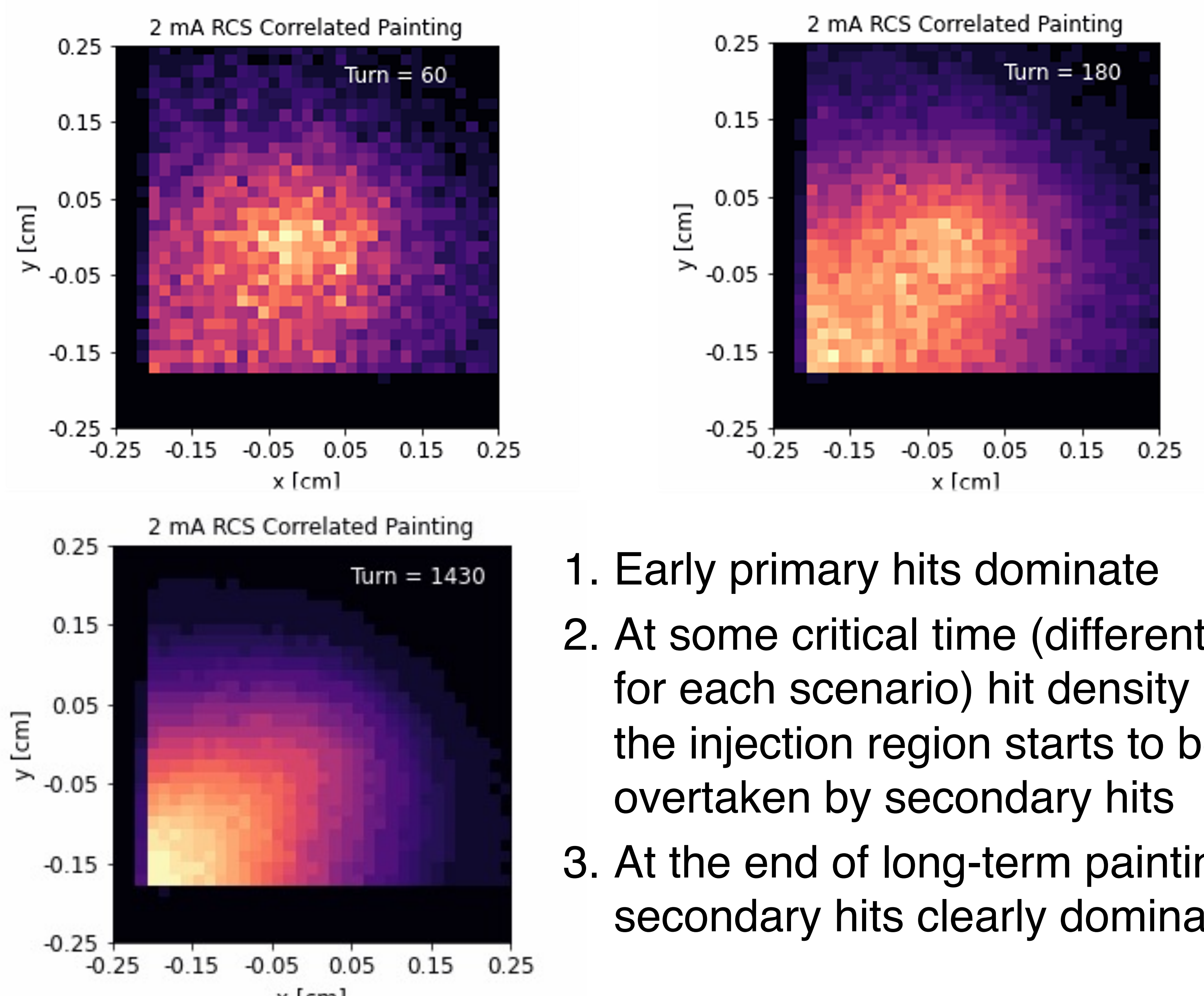

1. Early primary hits dominate

2. At some critical time (different for each scenario) hit density at the injection region starts to be overtaken by secondary hits

3. At the end of long-term painting secondary hits clearly dominate 